\title{
Recurrent focal segmental glomerulosclerosis after kidney transplantation: response to comments by Straatmann and Vehaskari
}

\author{
Howard Trachtman ${ }^{1} \cdot$ Rebecca Trachtman ${ }^{1}$
}

Received: 2 October 2015 /Revised: 2 October 2015 / Accepted: 19 October 2015 /Published online: 3 March 2016

(C) IPNA 2015

Dear Editor:

We thank Straatmann and Vehaskari [1] for correcting any misrepresentation of their work [2,3]. Their data strongly support the use of plasmapheresis to treat recurrent FSGS. Hopefully, a randomized clinical trial can be implemented to address this important problem.

\section{References}

1. Straatmann C, Vehaskari M (2016) Comments on Trachtman et al.: Recurrent focal segmental glomerulosclerosis after kidney transplantation. Pediatr Nephrol. doi:10.1007/s00467-015-3244-x

2. Trachtman R, Sran SS, Trachtman H (2015) Recurrent focal segmental glomerulosclerosis after kidney transplantation. Pediatr Nephrol 30:1793-1802. doi:10.1007/s00467-015-3062-1

3. Straatmann C, Kallash M, Killackey M, Iorember F, Aviles D, Bamgbola O, Carson T, Florman S, Vehaskari MV (2014) Success with plasmapheresis treatment for recurrent focal segmental glomerulosclerosis in pediatric renal transplant recipients. Pediatr Transplant 18(1):29-34

Howard Trachtman

howard.trachtman@nyumc.org

1 NYU Langone Medical Center, 403 E 34th Street,

New York, NY 10016, USA 